\title{
Multiple Raised White Plaques in the Right Colon
}

\author{
Anahita Sadeghi ${ }^{1}$, Behrouz Navabakhsh ${ }^{1}$, Hiva Saffar ${ }^{2}$, Mehdi Mohamadnejad $^{3, *}$
}

1. Digestive Disease Research Institute, Shariati hospital, Tehran University of Medical Sciences, Tehran, Iran

2. Department of Pathology, Tehran University of Medical Sciences, Tehran, Iran

3. Liver and pancreatobilliary Disease Research Center, Digestive Disease Research Institute, Tehran university of Medical sciences, Tehran, Iran.

\section{* Corresponding Author:}

Mehdi Mohammadnejad, MD

Liver and Pancreatobiliary Center, Digestive Disease Research Institute, Tehran University of Medical Sciences, Tehran, Iran. Tel: + 982182415104 fax: + 982182415400 Email: mehdi.nejad@gmail.com

Received: 30 May. 2019 Accepted: 20 Aug. 2019

\section{Please cite this paper as:}

Sadeghi A, Navabakhsh B, Saffar H, Mohamadnejad M. Multiple Raised White Plaques in the Right Colon. Middle East J Dig Dis 2019;11:234-236. doi:10.15171/mejdd.2019.155.

A 52-year-old woman was referred to endoscopy unit for colorectal cancer screening. Colonoscopy showed multiple white plaques, dispersed with normal mucosa in the ascending colon and cecum. Other parts of the colon were normal (figure. 1). Biopsy samples were taken and the histopathological examination confirmed the endoscopist's diagnosis (figures 2, and 3).
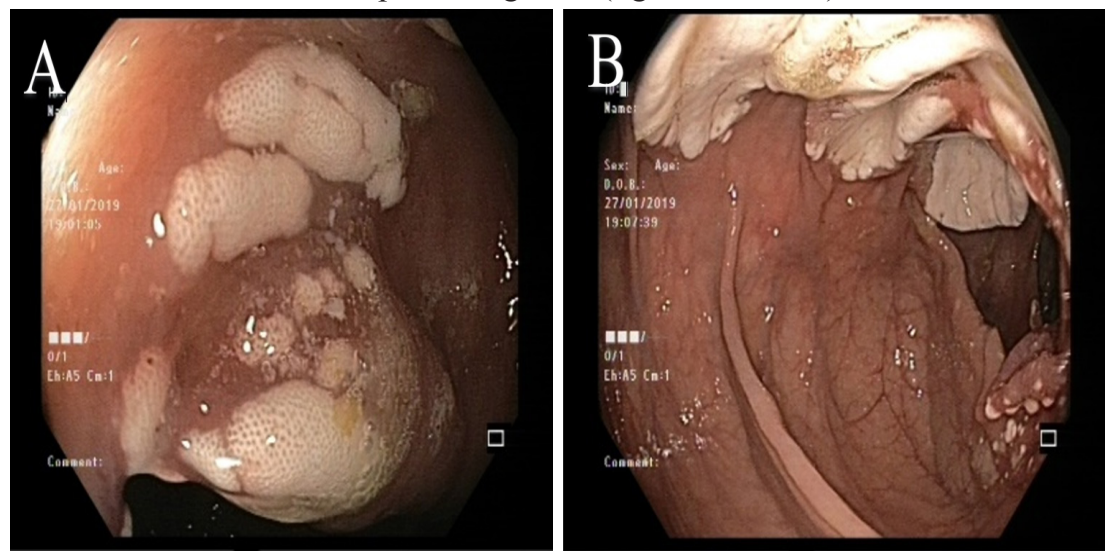

Fig.1: Colonoscopy: White plaques of the ascending colon adjacent to the cecum (A and B).
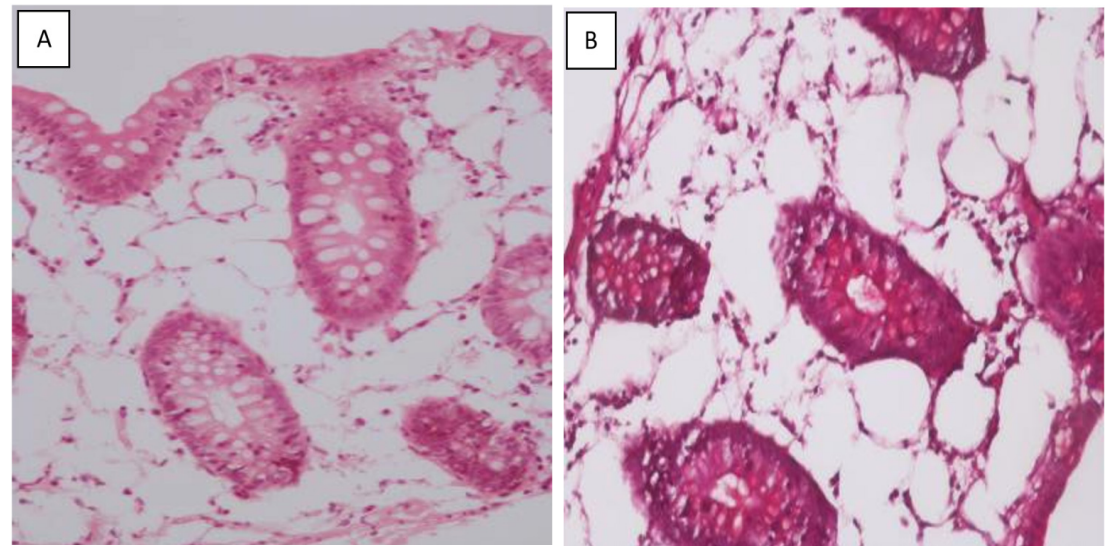

Fig.2: A- Biopsy of the colon mucosa showing vacuoles in lamina propria (H\&E stain). B-The vacuoles are negative for mucin (PAS staining) 


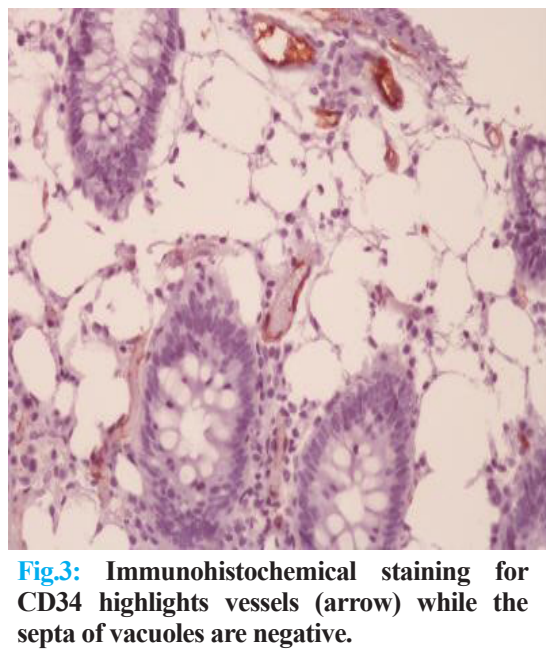

What is your diagnosis?

Answer

Colonic pseudolipomatosis

Histological examination of multiple colonic mucosal biopsy samples revealed Periodic acid-Schiff (PAS) negative (negative for mucin) vacuoles in the lamina propria of the colon, which is diagnostic for mucosal pseudolipomatosis. The septa of vacuoles were negative on immunohistochemical (IHC) staining for CD34 (compare with the positive endothelial lining in figure 3 ).

Colonic pseudolipomatosis is a relatively rare incidental finding of multiple occasionally confluent white plaques containing microbubbles reported in $0.02 \%$ to $1.7 \%$ of colonoscopy series. ${ }^{1}$ The endoscopic appearance is characteristic and has been named the snow-white sign or frost sign. ${ }^{2}$ On histological examination, intramucosal gas vacuoles trapped in the lamina propria is observed. This condition has been reported in endoscopic evaluations of the colon, stomach, and duodenum. ${ }^{3,4}$ Colonic pseudolipomatosis must be distinguished from pseudomembranous colitis, colonic lipomatosis, and malakoplakia. The major difference between a lipoma and a pseudolipoma is in the absence of adipocytes (ie, the absence of fat). Fat stains are consistently negative in mucosal pseudolipomatosis. Medical literature does not suggest a predilection for a specific age or sex in the prevalence of reported cases of colonic pseudolipomatosis. ${ }^{5}$

Snover and colleagues were the first who distinguished pseudolipomatosis from lipomatosis by refuting the presence of adipocytes through histochemical and ultrastructural analyses in $1985 .{ }^{6}$ Since then, multiple putative mechanisms have been proposed but the definite physio-pathogenesis remains elusive. One theory views pseudolipomatosis as a mechanical complication related to air pressure during colonoscopic air inflation similar to pneumatosis intestinalis. ${ }^{7}$ A possible histological dichotomy has been suggested by Nakosono and co-workers for pseudolipomatosis lesions. One subtype is described as uniformly sized vacuoles in the upper portion of lamina propria without involvement of submucosa and lymph follicles while the other subtype has more variation in the size of air vacuoles and is mainly seen in the lower portion of lamina propria and occasionally submucosa and is related to lymph follicles with a predilection for involvement of ileocecal valve area. The pathogenesis of the second subtype could be identical to pneumatosis coli, resulting from the penetration of gas into the mucosa through the crypts and possibly transecting the muscularis mucosa through the lymph follicles. ${ }^{1,8}$ Although muscularis mucosa and submucosa were not present in our patient's biopsy specimens, the presence of same-sized vacuoles is more consistent with the first histological subtype.

Other studies have suggested chemical colitis due to contamination of the endoscope's air-water channel with peracetic acid, hydrogen peroxide, or other peroxide containing agents and the ensuing chemical colitis as a possible causative explanation. These cases have been reported as epidemic clusters following some change in the disinfection routine of the endoscopy unit and the lesions seemed to spread while the colonoscopy was being performed. ${ }^{4,9,10}$ Since we have not observed an epidemy of similar cases and the disinfection routine has not been altered recently, this theory seems not to pertain to our case.

Because of its relative rarity, some endoscopic practitioners may be unacquainted with the highly characteristic endoscopic appearance of colonic mucosal pseudolipomatosis and it may be confused with other lesions such as pseudomembranous colitis, colonic lipomatosis, or malakoplakia. By this report we hope to help raise awareness of this self-limiting condition and its endoscopic and pathological features.

\section{Informed Consent:}

Written informed consent was obtained from the patient who participated in this study. 


\section{Financial Disclosure:}

The authors declared that this study has received no financial support.

\section{ETHICAL APPROVAL}

There is nothing to be declared.

\section{CONFLICT OF INTEREST}

The authors declare no conflict of interest related to this work.

\section{REFERENCES}

1. Iwamuro M, Tanaka T, Kawabata T, Sugihara Y, Harada $\mathrm{K}$, Hiraoka S, et al. Pseudolipomatosis of the Colon and Cecum Followed by Pneumatosis Intestinalis. Intern Med 2018;57:2501-4. doi: 10.2169/internalmedicine.0730-17.

2. Lapeyre B. The "frost sign" and the "snow white sign": intramucosal air injection or peroxide colitis? Endoscopy 2005;37:679. doi: 10.1055/s-2005-861332.

3. Stebbing J, Wyatt JI. Gastric 'pseudolipomatosis'. Histopathology 1998;32:283-4. doi: 10.1046/j.13652559.1998.0372h.x.

4. Cook DS, Williams GT. Duodenal 'pseudolipomatosis'. Histopathology 1998;33:394-5.

5. Ersoz F, Toros AB, Cakar E, Gunes ME, Culcu S, Sari S, et al. Colonic mucosal pseudolipomatosis: Are we aware of it? Ulus Cerrahi Derg 2015;32:90-2. doi: 10.5152/ UCD.2015.2886.

6. Snover DC, Sandstad J, Hutton S. Mucosal pseudolipomatosis of the colon. Am J Clin Pathol 1985;84:575-80. doi: 10.1093/ajcp/84.5.575.

7. Waring JP, Manne RK, Wadas DD, Sanowski RA. Mucosal pseudolipomatosis: an air pressure-related colonoscopy complication. Gastrointest Endosc 1989;35:93-4. doi: 10.1016/s0016-5107(89)72716-4.

8. Nakasono M, Hirokawa M, Muguruma N, Okamura S, Ito $\mathrm{S}$, Iga A, et al. Colonic pseudolipomatosis, microscopically classified into two groups. J Gastroenterol Hepatol 2006;21:65-70. doi: 10.1111/j.1440-1746.2005.03973.x.

9. Bahaa B, Hussain I, Sami A, Shahad MA, Fatma A. Ahmed HA. Colonic Mucosal Pseudolipomatosis: Diagnosis and Etiology. Adv Res Gastroentero Hepatol 2017;2:555596. doi: 10.19080/ARGH.2017.02.555596.

10. Jonas G, Mahoney A, Murray J, Gertler S. Chemical colitis due to endoscope cleaning solutions: a mimic of pseudomembranous colitis. Gastroenterology 1988;95:1403-8. doi: 10.1016/0016-5085(88)90380-0. 